\title{
Existing offset assignments are near optimal for an industrial AFDX network
}

\author{
Xiaoting Li, Jean-Luc Scharbarg, Christian Fraboul \\ INP-ENSEEIHT IRIT, Université de Toulouse \\ Toulouse, France \\ \{Xiaoting.Li, Jean-Luc.Scharbarg, Christian.Fraboul\}@enseeiht.fr
}

\author{
Frédéric Ridouard \\ LISI-ENSMA, University of Poitiers \\ Poitiers, France \\ frederic.ridouard@ensma.fr
}

\begin{abstract}
Avionics Full DupleX Switched Ethernet (AFDX) has been developed for modern aircraft such as Airbus 380. Due to the non-determinism of switching mechanism, a worst-case delay analysis of the flows entering the network is a key issue for certification reasons. Up to now most existing approaches (such as Network Calculus) consider that all the flows are asynchronous and they do not take into account the scheduling of flows generated by the same end system. It is then pessimistic to take into account such a synchronous scenario. Each end system can be considered as an offset free system, thus the main objective of this paper is to evaluate existing offset assignments in the context of an industrial AFDX network. Existing offset assignments are adapted to take into account specific characteristics of an AFDX network. Worst-case delay results are obtained according to these offset heuristics. It is shown that some existing heuristics are not efficient while some are near optimal for the studied industrial AFDX network.
\end{abstract} lay

Keywords-AFDX network, offset assignment, worst-case de-

\section{INTRODUCTION}

Avionic Full DupleX Switched Ethernet (AFDX [1]) has been proposed in order to satisfy the growing requirements of avionics application. Such a network is defined based on static network configuration and routing. The demonstration of a determined upper bound for end-to-end (ETE) communication delays on such a real-time network plays a key role. Different methods [2]-[5] have been presented for the worstcase delay analysis on the AFDX network. Among them, the Network Calculus [6] has been used for the certification of Airbus 380.

Since each end system of the AFDX network schedules its flows according to a local clock, it is pessimistic to consider that all frames arrive simultaneously (synchronous scenario) on this network. This issue has been addressed in [7], in which a computation method integrating the offsets of flows based on the Network Calculus approach has been developed. However, only one offset assignment originally designed for the CAN network in [8] was applied to an industrial AFDX network. It is interesting to consider other existing offset assignments [9], [10] in order to find the best algorithm for an industrial AFDX network. Moreover, the existing algorithms can be adapted in order to take into account specific characteristics of an AFDX network.
The objective of this paper is to evaluate and compute offset assignment algorithms for an industrial AFDX network. The goal of the evaluation is to measure the gap between offset assignment based on heuristics and the optimal assignment, which is intractable on an industrial AFDX network. An upper bound on this gap is computed, based on an optimal scenario.

This paper is organized as follows. Section II shortly introduces the context of the studied industrial AFDX network and existing offset assignments. Section III derives an ideal offset assignment which gives an optimal scenario for the scheduled flows. In Section IV, new heuristics integrating the AFDX characteristics are proposed. The existing and proposed offset assignments are applied to the industrial AFDX network, and their results are compared and analyzed in Section V. Section VI concludes and indicates directions for future research.

\section{CONTEXT}

\section{A. Introduction of the industrial AFDX network}

An AFDX network [1] is composed of end systems and switches. The inputs and outputs of the AFDX network, called end systems $(E S)$, are connected by several interconnected AFDX switches. Each end system can be connected to only one port of an AFDX switch and each port of an AFDX switch can be connected at most to one end system. Links between switches work in full-duplex mode.

A Virtual Link $(V L)$ standardized by ARINC-664 is a concept of virtual communication channel, which statically defines the flows. A connection defined by a Virtual Link is unidirectional, including one source end system and one or more paths leading to different destination end systems (multicast nature). A VL is characterized by:

- Bandwidth Allocation Gap $(B A G)$, the minimum delay between two consecutive frames of corresponding VL ranging in powers of 2 from $1 \mathrm{~ms}$ to $128 \mathrm{~ms}$, and

- $S_{\min }$ and $S_{\max }$, the minimum and maximum frame length which respect the standard Ethernet frame.

An AFDX network architecture is illustrated by Figure 1. According to this architecture, there are five end systems and two AFDX switches. On the example, $v_{1}$ has a unique path $\left\{e_{1}-S_{1}-S_{2}-e_{4}\right\}$ and $v_{5}$ has multi-paths $\left\{e_{3}-S_{2}-e_{4}\right\}$ and $\left\{e_{3}-S_{2}-e_{5}\right\}$. 


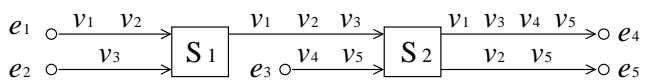

Figure 1. Example of an AFDX configuration

The industrial AFDX network interconnects aircraft functions in the avionics domain. It is composed of two redundant networks. Each network includes 123 end systems, 8 switches, 984 Virtual Links and 6412 VL paths (due to VL multicast characteristics). The left part in Table I gives the dispatching of VLs among BAGs. The right part in

\begin{tabular}{|c|c|}
\hline $\begin{array}{c}\text { BAG } \\
(\mathrm{ms})\end{array}$ & $\begin{array}{c}\text { Number } \\
\text { of VL }\end{array}$ \\
\hline 2 & 20 \\
4 & 40 \\
8 & 78 \\
16 & 142 \\
32 & 229 \\
64 & 220 \\
128 & 255 \\
\hline
\end{tabular}

\begin{tabular}{|c|c|}
\hline $\begin{array}{c}\text { Frame length } \\
\text { (bytes) }\end{array}$ & $\begin{array}{c}\text { Number } \\
\text { of VL }\end{array}$ \\
\hline $0-150$ & 561 \\
$151-300$ & 202 \\
$301-600$ & 114 \\
$601-900$ & 57 \\
$901-1200$ & 12 \\
$1201-1500$ & 35 \\
$>1500$ & 3 \\
\hline
\end{tabular}

Table I

BAGS AND FRAME LENGTHS

Table I gives the dispatching of VLs among frame lengths, considering the maximum length $S_{\max }$. The majority of VLs considers short frames. Table II shows the number of VL paths per length (i.e. the number of crossed switches).

\begin{tabular}{|c|c|}
\hline Nb of crossed switches & Number of paths \\
\hline 1 & 1797 \\
2 & 2787 \\
3 & 1537 \\
4 & 291 \\
\hline \multicolumn{2}{|c|}{ Table II } \\
VL PATHS LENGTHS
\end{tabular}

This industrial AFDX network works at $100 \mathrm{Mb} / \mathrm{s}$ and the technological latency of an AFDX switch is $16 \mu \mathrm{s}$. The overall workload (utilization) of the industrial network is about $10 \%$. Actually, the industrial AFDX network is lightly loaded in order to guarantee that buffers will never overflow. Both sporadic VLs and periodic VLs exist on the AFDX network, and offsets can be assigned to periodic VLs. There is no global clock in an AFDX network. Consequently, frame releases of different end systems are independent. However, each end system schedules its flows. This scheduling can be integrated in the worst-case delay analysis thanks to offsets. The next paragraph gives an overview of existing offset assignments.

\section{B. Existing offset assignments}

The offset assignment has been studied in [9] in the context of periodic task sets executed in a uniprocessor. Each task $\tau_{i}$ is characterized by a period $T_{i}$, a hard deadline $D_{i}$, a processing time $C_{i}$ and an offset $O_{i}$. In the context of uniprocessor, the systems can be classified into three classes in terms of offset:

- Synchronous system: all the tasks have the same fixed offsets, i.e., at time 0 , all the tasks generate one request;

- Asynchronous system: an offset is allocated to each task due to application constraints;

- Offset free system: any offset can be allocated to each task in order to improve the system schedulability.

For the third class, a key point is the choice of an offset assignment. The number of possible offset assignments is exponential.

In [9], an optimal offset assignment is proposed to exhaust all possible non-equivalent offset assignments. Although this method reduces significantly the number of combinations, the number remains exponential. Dissimilar offset assignment, denoted $G C D$, is then defined in order to reduce computational complexity in the comparison with the optimal offset assignment by providing a single offset assignment for a task set. This method tries to move from the synchronous case as much as possible. It considers a minimal distance $\left\lfloor\frac{\operatorname{gcd}\left(T_{i}, T_{j}\right)}{2}\right\rfloor$ between two requests of $\tau_{i}$ and $\tau_{j}$, where $\operatorname{gcd}\left(T_{i}, \stackrel{2}{T}_{j}\right)$ is the greatest common divisor of $T_{i}$ and $T_{j}$. This method treats task pairs $\left(\tau_{i}, \tau_{j}\right)$ by decreasing value of $\operatorname{gcd}\left(T_{i}, T_{j}\right)$.

Near-optimal offset assignment heuristics are derived in [10] based on the study of $G C D$. This assignment considers four alternative offset allocations when $G C D$ fails to generate a schedulable asynchronous situation. Since both these two approaches assign offsets to VLs pair by pair, they are called PairAssign in this paper. Besides the decreasing value of $\operatorname{gcd}\left(T_{i}, T_{j}\right)$, other heuristics are proposed considering criteria like utilization rate, i.e., $\frac{C_{i}}{T_{i}}$, and the value of $-\operatorname{gcd}\left(T_{i}, T_{j}\right)$ to decide the order of flow pairs. These four heuristics are denoted and defined as follows:

- RateAdd: $\frac{C_{i}}{T_{i}}+\frac{C_{j}}{T_{j}}$,

- $R A G C D:\left(\frac{C_{i}}{T_{i}}+\frac{C_{j}}{T_{j}}\right) \times \operatorname{gcd}\left(T_{i}, T_{j}\right)$,

- $R M G C D: \max \left(\frac{C_{i}}{T_{i}}, \frac{C_{j}}{T_{j}}\right) \times \operatorname{gcd}\left(T_{i}, T_{j}\right)$;

- GCDMinus: $-\operatorname{gcd}\left(T_{i}, T_{j}\right)$;

In [8], the authors addressed that the offset assignments mentioned above are not efficient when applied to the scheduling of automotive message, and an offset assignment algorithm is tailored for automotive CAN network. This algorithm, called SingleAssign in this paper, aims at choosing offsets to maximize the distance between frames. For $n$ flows emitted by one source node, sort them by increasing value of their periods and calculate $T_{\max }=\max _{i \in[1, n]}\left\{T_{i}\right\}$. The assignments start with the flow having smallest period and process one flow after another. For a flow $\tau_{k}(k \in[1, n])$, its offset $O_{k}$ is decided as follows:

- first search for the least loaded interval in $\left[0, T_{k}\right)$;

- then set $O_{k}$ in the middle of this interval;

- finally record all the frames of $\tau_{k}$ released in $\left[0, T_{\max }\right)$. 


\section{OPTIMAL SCENARIO OF SCHEDULED FLOWS OVER THE AFDX NETWORK}

Considering an industrial AFDX configuration with about 1000 flows, the optimal offset assignment proposed in [9] is intractable. Thus approaches based on heuristics have to be used. Then, the evaluation of the gap between the optimal offset assignment and the assignment generated by each heuristics is an important issue. For a given flow, this gap can be defined as the difference between the worst-case ETE delays obtained by, on the one hand considering the optimal offset assignment, on the other hand considering the offset assignment based on a heuristic. On a whole configuration, the gap is the average of the gaps obtained for the flows. Obviously, it is not possible to compute the gap for the optimal offset assignment on an industrial AFDX configuration, since the optimal offset assignment is intractable. Then, a first idea is to compute an upper bound on this gap.

This upper bound can be obtained by considering an ideal offset assignment, which minimizes the worst-case ETE delay for all the flows. This ideal assignment may not exist for a given configuration, but it is sure that it gives worstcase delays which are not higher than the ones obtained by the optimal offset assignment. This ideal assignment, denoted IdealAssign, minimizes the maximum waiting delay of every frame in each output port it crosses. It corresponds to the following scenario:

- At its source ES, a frame $f_{i}$ of a VL $v_{i}$ is not delayed by any other frames emitted by the same ES, i.e., the frame $f_{i}$ is transmitted immediately after its release;

- At each switch output port of its path, the frame $f_{i}$ crosses VLs generated by several ESs. $f_{i}$ can be delayed by exactly one frame coming from each of these ESs. The frame with the largest size $S_{\max }$ is considered. The delay encountered by $f_{i}$ at each switch output port takes into account the serialization effect (i.e., two frames cannot be received at the same time from an input link, see [3] for details).

Indeed, since there is no common clock among the end systems, there is no relationship between the releases of two frames from different end systems. Consequently, there exist scenarios where the two frames arrive at their first common switch output port at the same time.

Let us illustrate this scenario on the example depicted in Figure 2. This sample network has 4 VLs $v_{1}$ and $v_{2}$ emitted by the ES $e_{1}$ as well as $v_{3}$ and $v_{4}$ emitted by the ES $e_{2}$. The network works at $100 \mathrm{Mb} / \mathrm{s}$. The temporal characteristics of each VL are listed in Table III.

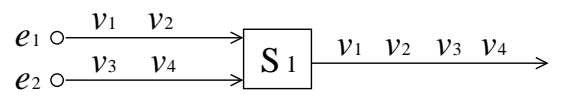

Figure 2. A sample AFDX network

\begin{tabular}{|c|c|c|c|}
\hline$v_{i}$ & $B A G_{i}(\mathrm{~ms})$ & $S_{\max _{i}}($ Byte $)$ & $C_{i}(\mu s)$ \\
\hline$v_{1}$ & 8 & 1000 & 80 \\
\hline$v_{2}$ & 8 & 1000 & 80 \\
\hline$v_{3}$ & 8 & 1000 & 80 \\
\hline$v_{4}$ & 8 & 500 & 40 \\
\hline
\end{tabular}

Table III

The Configuration of THE SAMPLE EXAMPLE IN Figure 2

The $\mathrm{VL} v_{1}$ is focused on. The IdealAssign leads to scenario illustrated in Figure 3 where the arrow represents the frame arrival of $\mathrm{VL} v_{i}, a_{i}^{h}$ is the frame arrival of $v_{i}$ at the node $h$, and the $\mathrm{i}$ means the transmission of a frame of VL $v_{i}$. At the ES $e_{1}$, the frame $f_{1}$ is transmitted as soon as it is released due to the separation from $v_{2}$. Since the ESs are not synchronized, at the output port of the switch $S_{1}$, the frame $f_{1}$ of $v_{1}$ can arrive at the same time as the frame $f_{3}$ of $v_{3}$ and it is delayed by $f_{3}$, i.e., $a_{1}^{S_{1}}=a_{3}^{S_{1}}$. Only one frame $\left(f_{3}\right)$ from the ES $e_{2}$ delays the frame $f_{1}$ at the output port of $S_{1}$ since $v_{3}$ and $v_{4}$ are separated far away from each other, and $f_{3}$ is considered due to the frame size $S_{\max _{3}}>S_{\max _{4}}$.

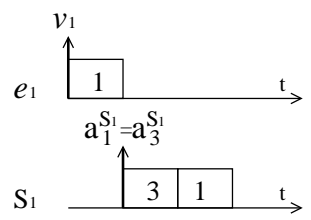

Figure 3. Scenarios of the VL $v_{1}$

The IdealAssign gives an upper bound on the reduction which can be obtained by an offset assignment algorithm. The next section proposes some offset assignment heuristics tailored for the AFDX network.

\section{OfFSET ASSIGNMENTS IN THE CONTEXT OF AFDX NETWORK}

In the context of a uniprocessor, a set of tasks shares a unique resource, i.e., the processor. The situation is different in the context of a switched Ethernet network, like the AFDX network, where a set of flows shares a set of output ports. Actually, each port is shared by a subset of all the flows. Consequently, the load can be different for each output port. The worst waiting time of a frame in an output port increases when the load of the output port increases. Then, it could be interesting to take into account the load of the output port in the offset assignment. This is illustrated in the example in Figure 4, where six VLs $v_{i}(i \in[1,6])$ are transmitted over the network. The temporal characteristics of each VL are given in Table IV. The network works at $100 \mathrm{Mb} / \mathrm{s}$ and the technological latency of switch is null.

The offset assignment SingleAssign is applied to this example network. The three VLs emitted by the ES $e_{1}$ are considered. The offsets are assigned to these three VLs in 


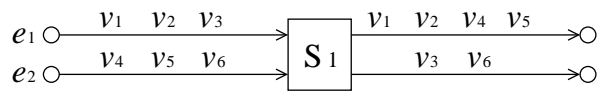

Figure 4. A small example of AFDX network

\begin{tabular}{|c|c|c|c|}
\hline$v_{i}$ & $B A G_{i}(\mu s)$ & $S_{\max _{i}}($ Byte $)$ & $C_{i}(\mu s)$ \\
\hline$v_{1}$ & 400 & 500 & 40 \\
\hline$v_{2}$ & 800 & 750 & 60 \\
\hline$v_{3}$ & 400 & 750 & 60 \\
\hline$v_{4}$ & 400 & 500 & 40 \\
\hline$v_{5}$ & 800 & 750 & 60 \\
\hline$v_{6}$ & 400 & 750 & 60 \\
\hline
\end{tabular}

Table IV

The Configuration OF THE SMALl EXAMPLE IN Figure 4

order: $O_{1}=0 \mu s, O_{3}=200 \mu s$ and $O_{2}=100 \mu s$. This case is drawn in part $e_{1}$ in Figure 5. Similar case at the end system $e_{2}$ is depicted in part $e_{2}$ in Figure 5. $v_{2}$ is focused on whose first frame $f_{2}$ is released at $O_{2}=100 \mu \mathrm{s}$. At the output port of the switch $S_{1}$ where $v_{2}$ visits, $v_{4}$ and $v_{5}$ from $e_{2}$ join the path of $v_{2}$ while $v_{3}$ has left. Then one possible scenario at this output port is depicted in part $S_{1}$ in Figure 5. It can be seen that when the frames $f_{1}$ and $f_{2}$ arrive at $S_{1}$, they are still separated far enough to avoid delaying each other. Similarly, the frames $f_{4}$ and $f_{5}$ from $v_{4}$ and $v_{5}$ are separated far enough when they arrive at $S_{1}$, consequently only one frame $f_{5}$ delays the studied frame $f_{2}$. Since the frame $f_{2}$ is released at the ES $e_{1}$ at time $\mathrm{O}_{2}=100 \mu \mathrm{s}$ and the transmission of frame $f_{2}$ is finished at the switch $S_{1}$ at time $280 \mu \mathrm{s}$, the delay of the frame $f_{2}$ is $R_{2}=280-100=180 \mu \mathrm{s}$.

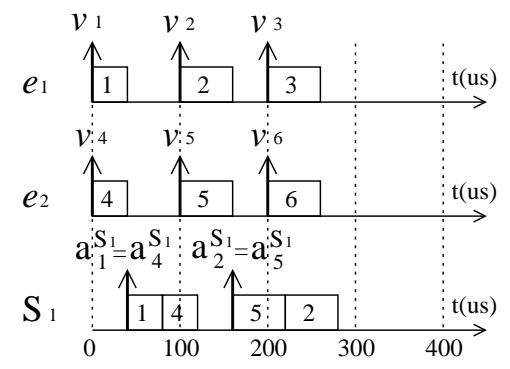

Figure 5. Illustration of the SingleAssign with low workload

The illustration in Figure 5 shows an example where the offset assignment SingleAssign succeeds to distribute the workload even in the output port of a switch. It is interesting to demonstrate the case when the workload increases. The example AFDX network in Figure 4 is under study and the maximum frame sizes of VLs $v_{1}$ and $v_{4}$ are increased to $S_{\max _{1}}=S_{\text {max }_{4}}=750$ Bytes $\left(C_{1}=C_{4}=60 \mu \mathrm{s}\right)$. According to the SingleAssign, the releases of frames at $e_{1}$ and $e_{2}$ are depicted in Figure 6 (same as in Figure 5). One possible scenario at the output port of $S_{1}$ is exhibited in part $S_{1}$ in Figure 6, where the studied frame $f_{2}$ finishes its transmission at time $300 \mu \mathrm{s}$. The delay of the frame $f_{2}$ is $R_{2}=300-100=200 \mu s$, higher than the case in Figure 5 $(180 \mu s)$. It increases due to the fact that when the frame $f_{2}$ arrives at $S_{1}$ at time $a_{2}^{S_{1}}=160 \mu \mathrm{s}$, the transmission of frame $f_{4}$, delayed by the transmission of frame $f_{1}$, is not completed which delays the transmission of frame $f_{2}$. For this case the SingleAssign could not separate frames at a crossed switch.

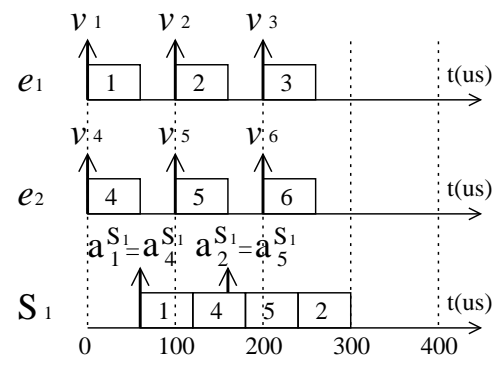

Figure 6. Illustration of the SingleAssign with high workload

Note that $v_{1}, v_{2}$ and $v_{3}$ emitted by $e_{1}$ visit three output ports: $e_{1}$ with the utilization $U_{e_{1}}=\sum\left(\frac{C_{1}}{T_{1}}+\frac{C_{2}}{T_{2}}+\frac{C_{3}}{T_{3}}\right)=$ 0.375 ; the upper output port of $S_{1}$ with the utilization $U_{S_{1}}=$ $\sum\left(\frac{C_{1}}{T_{1}}+\frac{C_{2}}{T_{2}}+\frac{C_{4}}{T_{4}}+\frac{C_{5}}{T_{5}}\right)=0.45$; and the lower output port of $S_{1}$ with the utilization $U_{S_{1}^{\prime}}=\sum\left(\frac{C_{3}}{T_{3}}+\frac{C_{6}}{T_{6}}\right)=0.3$. Consequently, for these three VLs, the most loaded port is the upper output port of $S_{1}$, followed by $e_{1}$ and the lower output port of $S_{1}$. We could first assign offsets to $v_{1}$ and $v_{2}$ which visit the most loaded port of $S_{1}$, then pass to the $v_{3}$, leading to the offsets: $O_{1}=0 \mu s, O_{2}=200 \mu s$ and $O_{3}=100 \mu s$. This case is illustrated in part $e_{1}$ in Figure 7. Similar case for the VLs emitted by $e_{2}$ is shown in part $e_{2}$ in Figure 7. Then one possible scenario for the frame $f_{2}$ at $S_{1}$ is identified in part $S_{1}$ in Figure 7, indicating that the delay of this frame is $R_{2}=380-200=180 \mu \mathrm{s}$, which is smaller than the one obtained by SingleAssign $(200 \mu s)$. The reason is that at the most loaded output port of $S_{1}$ the workload is further evenly distributed to reduce the waiting time in the buffer.

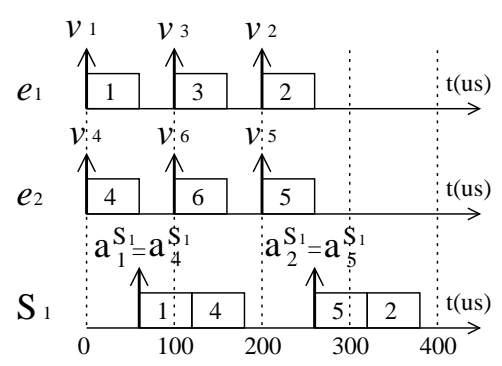

Figure 7. Illustration of the MostLoadSA with high workload

A proposed algorithm considers separating the VLs by 
decreasing utilization of the output ports they share. The offsets are first assigned to the flows visiting the most loaded port using the assignment SingleAssign, then to the flows which are not yet handled in the secondly most loaded port till all the flows of one ES are assigned with offsets. This algorithm is developed based on the assignment SingleAssign and denoted MostLoadSA.

For the PairAssign, a similar heuristic is proposed to consider the load of the output ports. This heuristic, denoted MostLoad, sorts the VL pairs $\left(v_{i}, v_{j}\right)$ by decreasing values of $L d_{i}+L d_{j}$, where $L d_{i}$ is the workload (utilization) of most loaded switch port crossed by $v_{i}$.

Due to the nature of the switched Ethernet, flows in one set can share several output ports. When flows share several common switches, the minimum interval between two frames decreases, which can increase the waiting time of a frame in the output port. Then the number of crossed switches can be considered in the offset assignments. For the PairAssign, a heuristic, denoted Crossed $S$, is proposed. It sorts the VL pairs $\left(v_{i}, v_{j}\right)$ by decreasing values of $c s\left(v_{i}, v_{j}\right)$, where $c s\left(v_{i}, v_{j}\right)$ is the number of common switches crossed by $v_{i}$ and $v_{j}$. For the SingleAssign, a similar heuristic, denoted CrossedSSA, is proposed which orders the VLs in one set by decreasing values of maximum number of crossed switch.

Besides the four new proposed heuristics, the existing offset assignment heuristics presented in Section II-B are applied to the AFDX network with the value of $B A G$ as the period. The evaluation on each offset assignment is processed in the next section.

\section{OBTAINED RESULTS}

The existing and proposed offset assignments introduced in Section IV are applied to the industrial AFDX network presented in Section II-A. In this evaluation, all the VLs are assumed to be strictly periodic. The computation is processed using the Network Calculus approach integrating the offsets, which has been developed in [7]. The computed ETE delay upper bounds of each offset assignment are compared with those obtained from the network without offset constraints. The statistic reductions on ETE delay upper bounds of each algorithm are listed in Table V. The columns Average, Max and Min give the average, maximum and minimum reductions, respectively.

The SingleAssign as well as its extended algorithms MostLoadSA and CrossedSSA outperform the PairAssign heuristics. Indeed, the average reductions obtained with the PairAssign heuristics are 23\% (GCD) and 32\% (RateAdd, RAGCD, RMGCD, GCDMinus, MostLoad and CrossedS). It is $49 \%$ for the SingleAssign and $51 \%$ for the SingleAssign based algorithms adapted to the AFDX network. On the considered example, the SingleAssign based algorithms are close to the IdealAssign, which gives an average reduction of $53 \%$.

\begin{tabular}{|c|c|c|c|}
\hline Heuristics & Average $\%$ & Max \% & Min \% \\
\hline IdealAssign & 53.48 & 83.29 & 21.00 \\
\hline GCD & 23.00 & 70.24 & 4.01 \\
\hline RateAdd & 32.89 & 73.50 & 5.08 \\
\hline RAGCD & 32.51 & 72.99 & 8.85 \\
\hline RMGCD & 32.29 & 70.77 & 9.99 \\
\hline GCDMinus & 32.95 & 70.06 & 8.83 \\
\hline MostLoad & 32.12 & 70.06 & 8.84 \\
\hline CrossedS & 32.32 & 73.03 & 8.90 \\
\hline SingleAssign & 49.67 & 83.29 & 18.84 \\
\hline MostLoadSA & 51.32 & 82.94 & 18.84 \\
\hline CrossedSSA & 51.29 & 82.94 & 18.84 \\
\hline
\end{tabular}

Table V

THE COMPARATIVE RESULTS

The PairAssign heuristics are not efficient in the studied context due to the limited different values of BAG, which lead to same values of $\operatorname{gcd}\left(B A G_{i}, B A G_{j}\right)$ for different VL pairs. Here is a small example in Figure 8. Considering VLs $v_{1}, v_{2}$ and $v_{3}$ with $B A G_{i}=4 \mathrm{~ms}(i \in[1,3])$ of $e_{1}$, there are three pairs: $\left(v_{1}, v_{2}\right),\left(v_{1}, v_{3}\right)$ and $\left(v_{2}, v_{3}\right)$. They have the same value of $\operatorname{gcd}\left(B A G_{i}, B A G_{j}\right)=4 \mathrm{~ms}(1 \leq i<j \leq 3)$. $G C D$ leads to $O_{1}=0 \mathrm{~ms}, O_{2}=O_{1}+\frac{\operatorname{gcd}\left(B \bar{A} G_{1}, B A G_{2}\right)}{2}=$ $2 \mathrm{~ms}$, and $O_{3}=O_{1}+\frac{\operatorname{gcd}\left(B A G_{1}, B A G_{3}\right)}{2}=2 \mathrm{~ms}\left(O_{2}=\right.$ $\left.O_{3}\right)$. The releases of the first frames for both $v_{2}$ and $v_{3}$ overlap, and the frames have to wait in the queue. This case is depicted in Figure 9.

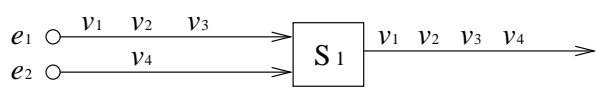

Figure 8. A small example of AFDX

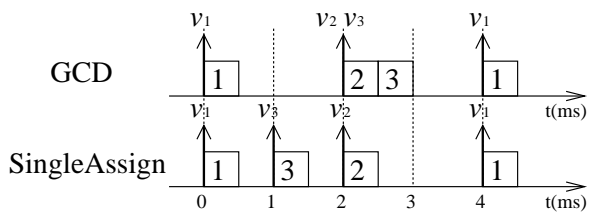

Figure 9. Comparison of GCD and SingleAssign

The situation is different when applying the offset assignment SingleAssign (Figure 9). With the same configuration, the offsets are set in order: $O_{1}=0 \mathrm{~ms}, \mathrm{O}_{2}=2 \mathrm{~ms}$ and $\mathrm{O}_{3}=1 \mathrm{~ms}$. In this way, no frame has to wait in the output queue of $e_{1}$.

The analyzed problem of $G C D$ for the industrial AFDX network exists for all the PairAssign heuristics because the computation of offsets mainly concerns the value of $\operatorname{gcd}\left(B A G_{i}, B A G_{j}\right)$ even if the order of pairs varies based on different criteria.

The results are further studied by a normalized method. For one path $\mathcal{P}_{x}$, the computed ETE delay upper bound without offset assignment is considered as the reference 
(denoted $r f_{x}$ ) and normalized as 100 . The computed result with one offset assignment (denoted $c p_{x}$ ) is taken as the comparison and normalized as $N c p_{x}$ :

$$
N c p_{x}=100+\left(\frac{c p_{x}-r f_{x}}{r f_{x}} \times 100\right)
$$

All the 6412 VL paths are sorted by increasing order of $N c p_{x}$. Three offset assignments are taken into account: IdealAssign, SingleAssign, and MostLoadSA. The comparative results are presented in Figure 10.

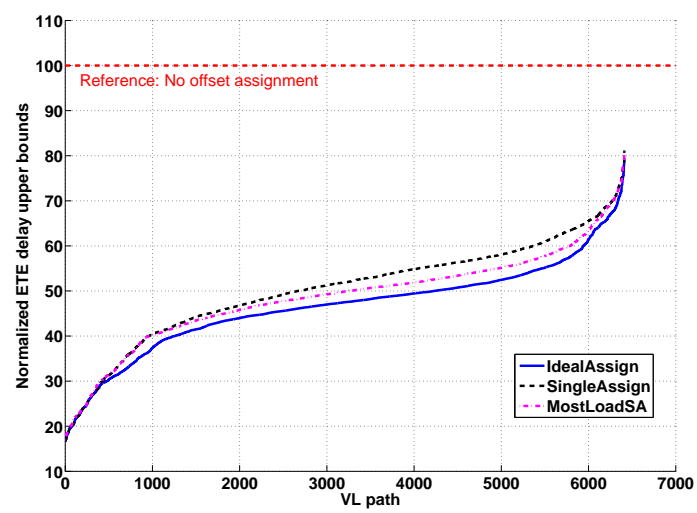

Figure 10. Comparative results of IdealAssign, SingleAssign and MostLoadSA

It can be seen in Figure 10 that the MostLoadSA curve is close to the IdealAssign curve, which reveals that this algorithm taking into account the AFDX properties works well on this industrial AFDX network. The gap between the SingleAssign curve and the IdealAssign curve is also small (although bigger than the gap with MostLoadSA curve). It suggests that a simple algorithm could be efficient to separate the flows of the industrial AFDX network.

Further evaluations have been conducted, leading to the same conclusions. They consider the same industrial AFDX architecture described in Section II-A and the overall workload $10 \%$ is kept. For each VL, the $S_{\min }$ and $S_{\max }$ are randomly chosen from 72 bytes to 1526 bytes, and the $B A G$ value is randomly chosen from $1 \mathrm{~ms}$ to $128 \mathrm{~ms}$ as the powers of 2 . The results show that the average ETE delay reduction brought by the IdealAssign is $45 \%$. The PairAssign heuristics bring average reductions ranging from $24 \%$ to $31 \%$, which are far from the IdealAssign. The algorithms based on the SingleAssign bring average reductions ranging from $39 \%$ to $40 \%$, which are closer to the IdealAssign.

\section{CONCLUSION}

In this paper, the offset assignments for the industrial AFDX network are studied. Since the optimal offset assignment is intractable in this context, an optimal scenario is built based on a presumed ideal assignment in order to upper bound the gap between the optimal offset assignment and each offset assignment heuristic. New heuristics considering the AFDX characteristics are proposed. Using the Network Calculus approach, the improvement on ETE delay upper bound bought by each heuristic is compared to the ideal algorithm. It is demonstrated that PairAssign heuristics are not efficient when applied to the industrial AFDX network due to the limited different values of $B A G$. The SingleAssign turns out a near optimal algorithm in the studied context. Although the heuristics integrating specific AFDX characteristics bring slight improvements in contrast to the SingleAssign, they are of increased complexity.

The industrial AFDX network considered in this paper is lightly loaded. The offset assignment for a switched Ethernet with heavier workload remains an open question, which is the subject of our ongoing work.

\section{REFERENCES}

[1] ARINC 664, ACCE Std. 664, 2002-2008.

[2] F. Frances, C. Fraboul, and J. Grieu, "Using network calculus to optimize the AFDX network," in Proc. 3rd Embedded Real Time Software Conference (ERTS'06), Toulouse, Jan. 2006.

[3] H. Bauer, J.-L. Scharbarg, and C. Fraboul, "Improving the worst-case delay analysis of an AFDX network using an optimized trajectory approach," IEEE Trans. Ind. Informat., vol. 6 , no. 4 , pp. 521-533.

[4] J.-L. Scharbarg, F. Ridouard, and C. Fraboul, "A probabilistic analysis of end-to-end delays on an AFDX avionic network," IEEE Trans. Ind. Informat., vol. 5, no. 1, pp. 38-49, Feb. 2009.

[5] H. Charara, J.-L. Scharbarg, J. Ermont, and C. Fraboul, "Method for bounding end-to-end delays on an AFDX network," in Proc. the 18th Euromicro Conference on Real-Time Systems (ECRTS'06), Germany, Jul. 2006, pp. 192-202.

[6] J.-Y. L. Boudec and P. Thiran, Network Calculus: A Theory of Deterministic Queuing Systems for the Internet, ser. Lecture Notes in Computer Science. Springer-Verlag, 2001, vol. 2050, iSBN: 3-540-42184-X.

[7] X. Li, J.-L. Scharbarg, and C. Fraboul, "Improving end-toend delay upper bounds on an AFDX network by integrating offsets in worst-case analysis," in Proc. IEEE International Conference on Emerging Technologies and Factory Automation (ETFA'10), Bilbao, Spain, Sep. 2010, pp. 1-8.

[8] M. Grenier, L. Havet, and N. Navet, "Pushing the limits of CAN - scheduling frames with offsets provides a major performance boost," in 4th European Congress on Embedded Real Time Software, Toulouse, France, Jan. 2008.

[9] J. Goossens, "Scheduling of offset free systems," Real-Time Systems, vol. 24, no. 2, pp. 239-258, Mar. 2003.

[10] M.Grenier, J. Goossens, and N. Navet, "Near-optimal fixed priority preemptive scheduling of offset free systems," in Proc. 14th International Conference on Real Time Network and Systems (RTNS'06), Poitiers, France, May 2006. 